\title{
Analysis of the formalin-fixed paraffin-embedded proteome: pitfalls, challenges and future prospectives
}

Evelyne Maes ${ }^{1,2,}{ }^{,}$, Valérie Broeckx ${ }^{1}$, Inge Mertens ${ }^{2,3}$, Xavier Sagaert ${ }^{4}$, Hans Prenen $^{5}$, Bart Landuyt ${ }^{1}$ and Liliane Schoofs ${ }^{1}$

${ }^{1}$ Research group of Functional Genomics and Proteomics, University of Leuven, Leuven, Belgium

${ }^{2}$ Flemish Institute for Technological Research (VITO), Mol, Belgium

${ }^{3}$ CFP-CeProMa, University of Antwerp, Antwerpen, Belgium

${ }^{4}$ Centre For Translational Cell \& Tissue research, Leuven, Belgium

${ }^{5}$ Department of gastro-enterology, Digestive oncology unit, University hospital Gasthuisberg, Leuven, Belgium.

*Corresponding author: evelyne.maes@bio.kuleuven.be

Postal address: Research group of Functional Genomics and Proteomics, KU Leuven, Naamsestraat 59, Leuven, Belgium Phone: +32 16323913 Fax: +32 16323902 


\section{Abstract}

Formalin-fixed paraffin-embedded (FFPE) tissues are a real treasure for retrospective analysis considering the amount of samples present in hospital archives, combined with pathological, clinical and outcome information available for every sample. Although unlocking the proteome of these tissues is still a challenge, new approaches are being developed. In this review, we summarize the different mass spectrometry platforms that are used in human clinical studies to unravel the FFPE proteome. The different ways of extracting cross linked proteins and the analytical strategies are pointed out. Also, the pitfalls and challenges concerning the quality of FFPE proteomic approaches are depicted. We also evaluated the potential of these analytical methods for future clinical FFPE proteomics applications. 


\section{Introduction}

In the post-genomic era, it has become clear that protein profile changes are important reflectors of biological and clinical phenomena. Furthermore, proteins are key effector molecules influencing pathological conditions. Mass spectrometry-based proteomics has therefore become an attractive technology to study disease-related protein differences. In disease related proteomics, the use of tissues is favorable over biological fluids, as investigating direct at the pathology site has several advantages, e.g. higher concentrations of disease-specific proteins. Although fresh or fresh frozen clinical specimens are ideal for proteomic analysis, the limited availability of these samples is a serious drawback. In recent years, it was suggested that formalin-fixed paraffin-embedded (FFPE) tissue might be a good alternative for frozen tissues (Nirmalan et al. 2008; Reimel et al. 2009; Klopfleisch et al. 2011).

Formalin fixation followed by paraffin embedding is the most common procedure for longterm preservation of clinical samples. Mostly, hundreds of formalin-fixed paraffinembedded diseased and paired healthy tissues are collected in hospital archives, as they are routinely prepared for pathological analysis. The by far most interesting issue of these FFPE specimens is that they hold pathological, clinical and outcome information inherently linked to every clinical sample. Also, large cohorts of samples might be available in a short period of time. Therefore, these samples are a real treasure for retrospective proteomic analysis, in order to elucidate pathological pathways or retrieve disease-associated biomarkers. However, the extensive formaldehyde-induced protein/DNA/RNA crosslinking 
is a barrier for many analytical platforms and successful removal of these crosslinks is complicated.

Today, several research groups try to unlock the proteome of FFPE tissues. In this review, we outline the use of FFPE samples in proteome analysis. We also describe the challenges and pitfalls concerning FFPE-centric proteomics approaches and conclude with future perspectives.

\section{Crosslinking and Protein extraction}

The fixation of clinical samples in formalin, followed by embedding in paraffin, is a worldwide known standard operating procedure used to perform histopathological examinations and to preserve clinical samples for a long time at room temperature. However, this processing of the tissue significantly modifies the proteins due to both fixation induced crosslinking events and exposure to heat and organic solvents (Ralton and Murray 2011). Typically, tissues are fixed for $24-48 \mathrm{~h}$ in a $10 \% \mathrm{v} / \mathrm{v}$ solution of formalin (= $37-$ $40 \% \mathrm{w} / \mathrm{w}$ formaldehyde in water with $10 \%$ methanol as stabilizer) (Nirmalan et al. 2008). As formaldehyde is the smallest aldehyde, it can quickly penetrate tissues and fix them by crosslinking proteins, RNA and DNA. These crosslinks are preferentially formed at primary amino groups and side chains of amino acids such as arginine, tyrosine, histidine, asparagine, glutamine and tryptophan. More detailed information about the chemistry of crosslinking can be found in the article by Klockenbusch et al. 2012 (Klockenbusch et al. 2012). Extracting proteins out of these crosslinked tissues requires robust methods to remove the crosslinks, but should not, on the other hand, produce changes itself. Until a decade ago, it was believed that the proteins in these samples were inaccessible for direct 
mass spectrometric analysis. However, since the introduction of the heat-induced antigen retrieval technique, developed to increase the reactivity in immunohistochemical assays in FFPE tissue, better extraction of proteins has been achieved (Shi et al. 1991). Based on this antigen retrieval procedure, Ikeda and co-workers were the first to enhance the extraction of proteins from FFPE tissue by heating the sections in a buffer containing $2 \%$ SDS (Ikeda et al. 1998). From then on, the quality and quantity of proteins that could be extracted was improved using customized buffer compositions. Although the QProteome FFPE Tissue kit (Qiagen, Hilden, Germany) and Liquid tissue MS protein prep kit (Expression pathology Inc., Rockville, MD) are commercially available and solubilization and isolation of intact proteins is feasible, many research groups produce their own extraction buffer in order to achieve even better results. Importance of heat, detergent, protein denaturant and physical agitation for efficient protein extraction are common components in all protocols. An overview of different buffer compositions can be found in table 1.

\section{Proteome analysis using mass spectrometry}

Mass Spectrometry (MS) based applications make it possible to analyze complex protein samples and to identify and/or quantify hundreds of proteins in a high throughput manner. Because MS allows the analysis of hundreds of proteins within a single assay and without the need of prior knowledge of potential proteins of interest, it has a major advantage over classical immunohistological assays. Also, there is no need to purchase commercially available antibodies or antibody development. 
In order to successfully analyze FFPE extracted proteins by mass spectrometry, reversal of these formalin-induced crosslinks is required and knowledge about the reaction products and the resulting modifications is needed. It is already known that the formation of modifications during fixation is influenced by several factors, including the rate of the crosslink reaction, the position and local environment of the amino acids, the $\mathrm{pH}$ of the reaction solvent as well as the components that are present and their concentrations (Metz et al. 2004). Several research groups tried to elucidate the reaction of formaldehyde with proteins and peptides (Metz et al. 2006; Metz et al. 2004; Toews et al. 2008). The reaction starts with the formation of methylol adducts $(\Delta m=+30)$ on primary amine groups. These adducts are followed by a partially dehydration which yields a labile Schiff base $(\Delta m=+12)$, that will form crosslinks with other amino acids. Although expected, both modifications are not found in raw MS data. Their results do also show that the chemistry of formaldehyde crosslinking is considerably more complex, and the resulting mixture of products is far more heterogeneous than first presumed. Therefore, these authors presume that crosslinked products might be difficult to assess by mass spectrometry.

However, besides the fact that some of these modifications are not yet elucidated, FFPE tissues are already used to perform proteomic analysis. Several research groups made the comparison between the proteome of fresh frozen material versus FFPE tissue, to evaluate the use of FFPE tissue as suitable sample source for proteomic analysis. These findings are nicely reviewed elsewhere (Tanca et al. 2012a). In short, an overlap of about $40 \%$ to $90 \%$ was found between the proteome profiles of fresh frozen versus FFPE tissue, depending on the analytical platform used. Also, the equivalence of the FFPE phosphoproteome and FFPE 
$\mathrm{N}$-glycome compared to fresh frozen tissue could be demonstrated using IMAC phosphopeptide enrichment in combination with LC-MS/MS (Ostasiewicz et al. 2010; Gamez-Pozo et al. 2011).

The use of FFPE tissue as sample source in retrospective clinical studies is widely applied today. Many different analytical proteomic platforms were used to achieve the same goal: find protein profiles which differ between diverse clinical conditions. These analytical platforms can be subdivided in four major categories: Shotgun (LC-MS/MS) proteomics, MALDI Imaging, gelbased platforms and targeted analysis of proteins. An overview of clinical proteome studies using human FFPE tissue is summarized according to analytical platform in Table 2 (Fig.1).

\subsection{Shotgun (LC-MS/MS) proteomics}

For a couple of years now, shotgun proteomics is the method of choice for the analysis of complex samples. Here, enzyme-digested proteins are separated using liquid chromatography (LC) and analyzed by tandem mass spectrometry (MS/MS). This peptidecentric gel-free analysis platform has the possibility to analyse samples in a high throughput manner and enables the identification and quantification of thousands of peptides in just a couple of hours.

In the context of FFPE proteomics, the first shotgun experiments were performed in order to identify as much confident peptides/proteins as possible, without any quantification purposes. Palmer-Toy and colleagues pioneered in 2005 in Reverse Phase (RP) LC-MS/MS proteomics of FFPE tissue and could identify 123 proteins in FFPE material. To identify these proteins, they prespecified the standard modifications, methionine oxidation and 
cysteine carbamidomethylation, but ignored other modifications induced by the crosslinking event. However, they tried to compensate this with an increased number of trypsin miscleavages and the use of the reversed database peptide strategy, i.e. translated open reading frames in reversed ('decoy') orientation, reducing false positive identifications (Palmer-Toy et al. 2005). Also in 2005, Prieto and colleagues identified 350 proteins in colon FFPE tissue using RP LC-MS/MS and in house developed software (Prieto et al. 2005). To gain insights in the pathology of prostate cancer, Hwang et al. provided a global proteomic analysis using the direct tissue proteomics method, in which the whole tissue slice is used to extract proteins, further digested with trypsin and separated applying RP LC-MS/MS (Hwang et al. 2007). For the confident identification of proteins, they prespecified methionine oxidation and phosphorylation (STY) as possible modification in combination with the decoy database searching approach to minimize false positive identification. However, again no special settings were used to handle with the crosslinked protein products. Bagnato and colleagues also used the Direct Tissue Proteomics (DTP) method, but compared it with Laser Capture Microscopy (LCM), which can isolate specific areas or cell types in a FFPE tissue. They found 710 proteins in coronary vessels FFPE tissue using the DTP strategy, but only 225 proteins were identified with more than one peptide identification. Using LCM in combination with in solution digestion and LC-MS/MS on the other hand, resulted in 495 multihit protein identifications (Bagnato et al. 2007). Again, no special settings were proposed to deal with FFPE tissue.

Crockett et al. downscaled the LC-system and used nano-RP-LC-MS/MS to achieve higher sensitivity. In their proof-of-principle experiment, they used the SUDHL-4 cell line, derived 
from human transformed B-cell lymphomas, pelleted the cells, fixed and embedded them and performed a shotgun proteomic analysis. To digest the extracted FFPE proteins, they used both trypsin and glutamic C endopeptidase, ensuring that some crosslinked proteins, inaccessible for trypsin digestion, could be digested and thus identified. A result of 324 unique protein identifications from 10 replicate runs was achieved by combining the identified proteins from trypsin digestion, glutamic $\mathrm{C}$ digestion and a combination of both enzymes. However, the authors did not specify any modifications using database search, probably explaining why the number of protein identifications is still limited (Crockett et al. 2005). Cheung and colleagues identified 523 unique proteins in pancreatic cancer using tryptic digestion in combination with triplicate nano-RP-LC-MS/MS runs (Cheung et al. 2008). Also here, no modifications were specified. Donadio and colleagues optimized extraction buffers containing high amounts of SDS for both gelbased and gelfree purposes and furthermore tried to use it for LC-MS/MS by combining 'SDS out kit' and TCA/Acetone precipitation. With this gelfree protocol, they were able to identify 163 confident proteins from adenoma parathyroid tissues using RP LC-MS/MS settings (Donadio et al. 2011). However, despite the effort they put in the efficient extraction of proteins, no solutions were proposed to deal with the protein crosslink problem for MS data analysis.

Since the development of the multidimensional protein identification technology (MudPIT) in 1999 (Link et al. 1999; Washburn et al. 2001), where complex samples were first separated using strong cation exchange $(\mathrm{SCX})$, and all the separate fractions are then analyzed using online reverse phase (RP) chromatography, the use of 2D-LC to elucidate complex proteomes has emerged exponentially. Also in the FFPE proteomics, 2D-LC was 
used in several different setups. The use of an integrated capillary isoelectric focusing (CIEF) and nano-RP-LC-MS/MS system was used to identify over 1800 confident proteins in renal FFPE samples (Shi et al. 2006). Although the authors did not mention any prespecified modifications, the increased number of identification compared to prior proteome experiments is stunning and results from the combination of 2D separation and nano-LC systems. This 2D-LC combination was also used by Guo and co-workers, who splitted the FFPE protein sample into 19 CIEF fractions and resolved them further by nanoRP-LC and nano-ESI-LTQ-MS/MS. Using this approach, they could identify 2,733 proteins in glioblastoma multiforme FFPE tissues (Guo et al. 2007). Concerning prespecified modifications, the authors made following settings: alkylated cysteine as fixed modifications and acetylation ( $\mathrm{N}$-terminus + lysine) and oxidized methionine as variable modifications. They also included the decoy strategy to outrule false positive identifications.

The combination of $1 \mathrm{D}$ gel electrophoresis and LC-MS/MS is another 2D experimental setup which is used by Tanca and colleagues to reduce the complexity of the proteome of neuroendocrine archival tissue (Tanca et al. 2011a). They compared two different fractionation methods: excision of 13 visible protein bands vs slicing of the whole gel lane into 38 fractions. Whole gel lane fractionation yielded more protein identifications (153) compared to visible band excision (92). Again, crosslinked protein products are ignored in the database searching methods, although they mention the fact that the position on 1D gels gives an indication of the proteins or their crosslinked complexes. 
In clinical FFPE proteomics however, most research groups perform proteome studies to find protein profiles which differ in abundance between diverse conditions. For quantification purposes in FFPE proteomics research, label-free approaches have become popular. In these label-free approaches, each sample is individually prepared and analyzed in an 1D or 2D LC-MS/MS setup. Furthermore, quantification can be achieved by protein abundance correlation through either mass spectrometric ion intensities of MS or MS/MS signals (peak intensity) or the number of MS/MS spectra per peptide and protein (spectral counting)(Li et al. 2012). This last method is more frequently been used in FFPE proteomics, as it suffers less from the peptide overlap of different fractions. Bateman and coworkers for example, used spectral counting to find differential protein profiles in breast cancer samples to indicate early-to-late stage progression as well as recurrence of the disease (Bateman et al. 2011). Also other research groups used FFPE proteomics in combination with spectral counting to elucidate protein expression changes involved in tumor progression. Patel and colleagues profiled the progression of head and neck cell squamous carcinoma at proteome level. In total, 391 proteins were identified in normal squamous epithelium, 866 in well differentiated epithelium, 729 in moderate differentiated and 676 in poorly differentiated epithelium (Patel et al. 2008). Also in renal cancer, spectral counting was used to characterize different stages using LC-MS/MS (Perroud et al. 2009). In the search for stage-related protein candidates in lung cancer, Kawamura used spectral counting and found more than 500 identified proteins, of which 81 were correlated with stage la or Illa (Kawamura et al. 2010). To understand the proteomic changes in melanoma progression and metastasis, Huang et al. used the label- 
free peak intensity approach and did find 120 proteins that were differentially expressed in metastasis compared to primary melanomas (Huang et al. 2009).

Also for quantitation purposes, the addition of an orthogonal LC dimension will help to mine deeper into the FFPE proteome. Naidoo and co-workers for example, used spectral counting in combination with MudPIT separation to assess proteomic differences between primary pancreatic tumors and matched lymph node metastasis (Naidoo et al. 2012). From the 115 proteins that were differentially expressed, S100P and 14-3-3 sigma were further confirmed using immunohistochemistry (Naidoo et al. 2012). Negishi and co-workers used their own developed label free method called 2DICAL (2 Dimensional Image Converted Analysis of Liquid Chromatography) to find differentially expressed proteins in tongue cancer. They could collect 25,018 MS peaks and did find 72 peaks which were significantly differentially expressed between cancer and normal epithelia (Negishi et al. 2009). The research group of Mathias Mann used specialized MaxQuant software to perform labelfree quantitation for proteins extracted from both colon and breast archival tissues, using Filter aided sample preparation - Strong Anion Exchange (FASP-SAX) fractionation, followed by RP separation coupled to an Orbitrap mass spectrometer (Wisniewski et al. 2011). Using the same strategy and multi-enzyme digestion, the research group revealed that identification of 10,000 proteins from human colon FFPE tissue is possible (Wisniewski et al. 2012).

All these studies indicate that these label-free techniques have potential to elucidate biological questions regarding differential protein profiles using archival FFPE tissues. The 
major advantage of this label-free technique is that no additional steps for labeling are needed and thus no extra costs are involved. Certainly in FFPE context, where additional labeling chemistry might complicate the results even more, label-free methods are popular. The major disadvantage however, is that run-to-run variation needs to be controlled to the most possible extend, which might be a challenge, even with state-of-theart instruments. Also, although a broad dynamic range is reached for peptide identification, a high amount of spectral counts is necessary to obtain reliable quantification results. Therefore, some authors opted to use enzymatic or chemical labeling strategies.

Hood and co-workers pioneered in labeling-based methods on FFPE tissue using enzymatically facilitated ${ }^{18} \mathrm{O}$ labeling to quantify tryptic peptides after separation using nano-RP-LC-MS/MS. They could identify 1,300 unique peptides from 702 proteins selected out of 200,000 cells of benign prostatic hypotrophy tissue and found 69 differentially expressed proteins between prostate cancer and benign prostatic hypertrophy (Hood et al. 2005). Although identified and quantified, prostate specific antigen (PSA) could not make a distinction between these two prostate-specific diseases. The ${ }^{18} \mathrm{O}$ proteolytic labeling strategy was also employed by Nazarian et al., to profile the FFPE proteome of brainstem glioma compared to normal tissue (Nazarian et al. 2008). In total, they could identify and quantify 188 proteins and found 54 up-regulated proteins, of which several were already linked to cancer. Although incubation of peptides with trypsin in ${ }^{18} \mathrm{O}$ enriched water provides a straightforward way to label peptides, a major disadvantage of this technique is that the reproducibility is heavily dependent on many factors including labeling time, 
temperature, amount and activity of the trypsin and also back reaction between ${ }^{18} \mathrm{O}$ and ${ }^{16} \mathrm{O}$ is possible (Wu et al. 2012).

For that reason, chemical labeling strategies e.g. Isobaric tags for relative and absolute quantification (iTRAQ) are also used. The major advantages using isobaric tags involve the possibility to multiplex up to 8 samples into one LC-MS/MS run thereby saving instrument time and reducing the run-to-run variation seen in label-free methods. Moreover, it has been shown that isobaric chemical labeling can provide more accurate and precise quantification of proteins compared to label-free methods (Li et al. 2012). A disadvantage, on the other hand, is that the labels are only integrated after protein reduction, alkylation and digestion, creating thus variability. In the context of FFPE proteomics, the labeling efficiency of crosslinked peptides is also major concern. Jain and co-authors though, demonstrated that the iTRAQ technology was able to quantify 114 FFPE extracted proteins, which could lead to discrimination between HIV(+) and HIV(-) patients (Jain et al. 2008). Xiao and co-workers used iTRAQ quantification in combination with MudPIT technology to perform a proteome analysis on nasopharyngeal carcinoma (Xiao et al. 2010). According to the author, a good ITRAQ labeling performance was achieved, despite the presence of crosslinked proteins. To elucidate the pathology of diabetic nephropathy, Nakatani et al. used ITRAQ and could find 55 proteins that were upregulated and 45 downregulated proteins in glomerular cross sections from diabetic patients with nephropathy. Most of them are linked to renal and urological disease (Nakatani et al. 2012). Again, no special adjustments were made to deal with the crosslinking problem. 


\subsection{Mass Spectrometry Imaging}

Mass spectrometry imaging (MSI) is a mass spectrometric technique that enables the visualization of proteins without losing spatial information. In proteomics applications mostly Matrix Assisted Laser Desorption Ionization (MALDI)-MSI is applied (Amstalden van Hove et al. 2010). In MALDI-MSI, matrix is first deposited on a thin tissue section, the sample surface is rastered according to a predefined rectangular $\mathrm{X}, \mathrm{Y}$ grid and full mass spectra are acquired in each pixel where analytes are deserted upon laser irradiation. The intensity values of different ions can be plotted upon the initial raster, generating a spatial image (Walch et al. 2008; Cornett et al. 2007). Furthermore, the technique allows to map hundreds of proteins/peptides simultaneously in a thin tissue section with a spatial resolution of $50 \mu \mathrm{m}$ (Minerva et al. 2012). A major advantage using imaging techniques is that no extensive extraction procedures, purifications and separations are conducted. Also, there is no need to develop specialized antibodies, which makes it an ideal approach for biomarker discovery. The nature of the proteins and their distribution in the tissues can be directly attributed to morphological or biochemical changes (Goodwin et al. 2008). Herefore, it is complementing classical immunohistological approaches, like immunohistochemistry and in situ hybridization, that require prior knowledge of the target proteins (Angel and Caprioli 2013). However, the MALDI-imaging method works best for small hydrophilic and abundant proteins but fails to detect a large number of proteins, even in fresh material. Also, absolute quantification of proteins using MSI is not possible yet (Angel and Caprioli 2013). 
The use of FFPE tissue slices as an alternative for fresh frozen material for MSI is known for several years now. In 2007, Lemaire and coworkers could show that direct tissue MSI of FFPE tissues stored less than 1 year was feasible for peptides smaller than 5 kDa using 2,4dinitrophenylhydrazine as matrix. To analyze the proteins in the FFPE sample, they implemented an enzymatic digestion protocol, as MSI imaging of FFPE tissues is mainly limited to peptide analysis, because chemical crosslinked proteins are difficult to assess with mass spectrometry (Lemaire et al. 2007). More recent reports illustrate that FFPE imaging of proteins/peptides by $\mathrm{MSI}$ is feasible by combining the antigen retrieval technique and in situ enzymatic digestion before matrix application (Gustafsson et al. 2010; Djidja et al. 2009; Seeley and Caprioli 2011; McDonnell et al. 2012).

The lab of Caprioli could show that the spatial expression of some proteins, including S100A9, differs between adenocarcinoma and squamous cell lung carcinoma by application of tissue microarrays (TMA) (Groseclose et al. 2008). In TMAs several tissue cores are placed into a single paraffin block, sectioned, and in this way analyzed simultaneously. TMAs have gained tremendous interest as these allow high throughput analysis from large clinical cohorts (Groseclose et al. 2008). In 2010, TMAs and MALDI MSI were applied to detect cancer specific or histological type-specific proteins using gastric cancer FFPE samples (Morita et al. 2010). Morgan and colleagues also combined TMA and MALDI MSI and found a peptide signature that could accurately distinguish malignant from normal renal tissue (Morgan et al. 2013). MALDI-MSI of FFPE tissues was also used to distinguish spitz nevus from spitzoid malignant melanoma. Five peptides were found to be differentially 
expressed and could classify spitz nevus with $97 \%$ sensitivity and $90 \%$ specificity using a validation cohort of 59 samples (Lazova et al. 2012).

\subsection{Gelbased proteomics}

Although gelbased proteomics is still the only technique which can visualize thousands of proteins in one image, interest in the workhorse of proteomics has decreased. Also in FFPE-applied proteomics, the number of research articles is limited. In 2003, Ahram and colleagues showed that the application of 2D gels to ethanol-fixed paraffin embedded tissues was more successful than using tissues fixed with formaldehyde (Ahram et al. 2003). The ethanol fixed protein spots in the gel were a bit fuzzy, but a good comparison with the frozen material was possible. The 2D gel separation of FFPE extracted proteins on the other hand, did not show any spot, only streaking and blurring. In 2009, Ono and coworkers did use 2D DIGE to compare the FFPE proteome of uterine cervix squamous cell carcinoma and healthy tissues, but could only show a low-quality 2D map with smears (Ono et al. 2009). Donadio and colleagues applied 2D electrophoresis using FFPE material from patients with sporadic primary hyperparathyroidism and did only found a low quality pattern of 16 spots and smears (Donadio et al. 2011). Until now, the research groups of Alessandro Tanca and Maria Filippa Addis are the only one managing to successfully separate proteins extracted from FFPE tissues using 2D gel electrophoresis (Addis et al. 2009; Tanca et al. 2011b; Tanca et al. 2012c). Although their patterns are more comparable with fresh frozen tissues than other research groups ever achieved, the quality of FFPE 2D gels remains questionable. 


\subsection{Targeted proteomics}

Selected Reaction Monitoring (SRM), also called Multiple Reaction Monitoring (MRM), is a technology that also complements the shotgun proteomics strategy as it allows the quantification of a predefined set of proteins. In an SRM workflow, the mass spectrometer will first target a specific peptide of interest in a first stage followed by targeting one (SRM) or more (MRM) of its fragment ions in a second stage. The combination of the precursor ion and fragment ions allows to specifically target the protein of interest in different samples, as all other acquired signals can be ignored (Picotti and Aebersold 2012; Lange et al. 2008).

The performance characteristics of FFPE extracted protein quantitation by MRM is assessed by Sprung and colleagues. Their results did show that, as expected, the formalininduced chemical modifications decrease the sensitivity of the MRM measurements, meaning that fewer targets will be available for quantification compared to fresh tissues. However, in their proof-of-concept experiment in which they quantified the HER2 receptor expression profiles in breast tumors, the assay sensitivity of FFPE extracted proteins and fresh frozen samples was comparable, when using unmodified peptides (Sprung et al. 2012). Grüzel and co-workers also made use of MRM quantification in order to find preeclampsia related calcyclin peptides in placental FFPE tissue. Their MRM assay demonstrated that in pre-eclamptic patients elevated levels of calcyclin is observed in placental trophoblas cells compared to normal trophoblast cells (Guzel et al. 2011). An MRM quantitative analysis for stage-related proteins upon non-metastatic lung adenocarcinoma was performed by Nishimura and colleagues. This study suggested that 
napsin-A and anterior gradient protein 2 homolog (hAG-2) would be useful for determining stage IA or IIIA lung adenocarcinoma. Both proteins could also be related to metastasis (Nishimura et al. 2010). In the context of FFPE extracted proteins for MRM applications, the Absolute QUAntification (AQUA) technique is also introduced. In the AQUA workflow, a peptide containing a stable-isotope labeled amino acid is synthesized based on the sequence of the targeted peptide of interest. This synthesized 'heavy' peptide is then spiked into the complex proteome sample and used as internal standard for quantification (Ye et al. 2009). The research group of Han used the AQUA technique to quantify prostate specific antigen (PSA) in prostate FFPE tissues and did find a trend of increasing levels of PSA in more advancing tumors (Hwang et al. 2007). To quantify pyruvate kinase M2 in endometrial samples and to confirm overexpression in cancer, Desouza et al. used Tags for Relative and Absolute quantification (mTRAQ) (DeSouza et al. 2010). In mTRAQ, up to three non-isobaric labels can be used to label a known quantity of a synthetic peptide whose sequence is identical to the tryptic peptide of interest and on the other hand, the sample containing tryptic peptides. After mixing and LC separation, the labeled peptides provide unique MRM transitions, both in MS mode (three non-isobaric parent ions) and in MS/MS mode (non-isobaric sequence ions) (DeSouza et al. 2008).

\section{Pitfalls and challenges}

Although a vast archive of clinical samples is present, FFPE tissues have been considered as obstinate to proteomic analysis. The reason why molecular analysis of these tissues is often questioned, is through the many challenges and pitfalls one is confronted with. 
The presence of protein/DNA/RNA crosslinks is one of the most important issues which hamper proteomic analysis. Extraction of proteins from archival FFPE tissues is mostly accompanied by a combination of exposure to organic solvents, heat and proteolysis. Although shown to be efficiently, it even might be the question whether all proteins stay soluble in this combination. Also, the reversal of crosslinks renders several by-products, inaccessible for mass spectrometric analysis and possibly disturbing high quality LC separations. However, it is also known that only a small percentage of formaldehydereactive amino acids form irreversible chemical modifications. In vitro models do show that the crosslink event is more complicated than theoretically assumed, and thus more unspecified modifications will turn up. Also, predicted peptide modifications, like methylol derivatives, are often not detected in experimental data. In addition, modifications due to chemical crosslinking might hinder tryptic digestion in some extent.

This will lead to a second major challenge: the confident identification of proteins. Not only will unknown and unexpected modifications on (tryptic) peptides lead to a decreased protein and proteome coverage, also biases exist compared to fresh material in identified basic proteins and in the lysine/arginine ratio of identified proteins, as basic amino acids, and in particular lysine, are known to be involved in the crosslinking process (Tanca et al. 2012a). Therefore, new bio-informatic approaches are needed to unlock the full FFPE proteome. The development of new algorithmic data-analysis software for LC-MS/MS data could solve this problem to a major extent. 
A third challenge is related to working with human tissues. It is already known that the variability between human samples is partly due to genetic diversity of humans, different environments, but also differences like age, gender and race that need to be controlled whenever one wants to perform proteomic profiling studies. Also, some ethical issues need to be considered, such as permissions that must be obtained from the patient before using the clinical sample for scientific purposes. This might not be a problem for samples gathered these days, but must also be present for FFPE samples that are already stored for over 10 years.

Finally, several other, more technical factors determine the variability of FFPE proteome data. One of the major parameters that should be controlled is the time between the resection of the tissue and the fixation in formalin. Ideally, samples should be fixed immediately and completely from the living state. In animal models, this can be achieved using in vivo perfusion. In humans however, surgically removement is the only option, and anoxic periods due to anesthesia and surgical clamps can influence the expression patterns of proteins. These factors cannot be strictly controlled because they impact patient care, but could, on the other hand, be recorded in detail (Hewitt et al. 2008). Also the way of conservation of the tissue between resection and fixation (either dry or bading in a solution) can have implications and should be standardized.

Another source of variability (and probably the easiest to control) is the fixation procedure. Three aspects are important during this protocol: the thickness of the tissue, the volume of the fixative and the fixation time. Unsuccessful optimization of these 3 elements results in under- or overfixation of the clinical samples, resulting in higher variation between 
different specimens. Through the large diversity of size of resected specimens, fixation procedures might be difficult to standardize. Therefore, we suggest that larger specimens should be sectioned as soon as possible after their resection and the volume of the fixative is adapted to the size of the specimen, starting from a minimum formalin:tissue ratio is 10:1. Concerning different fixation times, Tanca and co-workers made an evaluation of the efficiency of protein extraction and the quality of the obtained quantitative data. Their results show that successful identification of peptides decreases, as the fixation time increases (Tanca et al. 2012b). Unfortunally, it is common practice to fix specimens overnight or even over the weekend. Failure to standardize these steps will thus pose problems to the researchers working with the material.

The archival time is also an important parameter that can introduce variability in datasets, as it influence the retrieval of proteins over time (Balgley et al. 2009). On top, differences in storage conditions e.g. temperature, humidity,... will also have an effect on the variation of the FFPE proteome. For data interpretation, the way of isolation of the tissue e.g. cutting cylindrical section, working with tissue slices or laser microdissected cells, will also influence the data. Overcoming all these technical issues and accomplish the goal of standardization is thus a major challenge.

\section{Future perspectives}

Recent advances in the field of FFPE proteomics demonstrate that unlocking the FFPE proteome might have major potential for retrospective and translational biomarker research. Unfortunately, current practices lack the required standardization. Therefore, 
the use of fresh tissue remains the gold standard. To establish the use of FFPE material as primary tissue source for proteome studies, more investigations are needed to reduce the numerous variables and to achieve the many challenges. In the first place, protocol refinement is needed to manage the confounding FFPE data analysis which arises from different fixation times, storage periods, incomplete crosslink reversal etc. Therefore, more standardization in procedures of fixation and storage are needed to reduce biases and to maximize the dependability of the obtained proteomic results. These standard operation procedures should be systematically used in every hospital with an FFPE archive, which should create the possibility to perform multicentric proteomic studies. We also recommend obtaining as much information as possible regarding the surgery parameters, fixation times etc., which can only improve the quality and reproducibility of the scientific research. Also, existence of measures to assess the sample quality of archival tissues, both at molecular and morphological level, is critical and should reduce the variability in degradation effects between samples. Finally, the unraveling of all the peptide modifications induced by formalin crosslinking by means of bioinformatics and understanding the full process of crosslinking, might lead the FFPE proteome research towards complete acceptance. This translational (biomarker) research strategy depends thus on the interdisciplinary contributions of clinicians, scientists and biostatisticians.

When evaluating different analytical platforms used in proteomics today, LC-MS/MS will probably have the most potential to unlock the FFPE proteome because of its capability to identify thousands of proteins in just a couple of hours. However, obtaining such results is only possible when new developments in the interpretation of FFPE raw data files succeed. 
Also the combination of LC-MS/MS and MALDI-MSI might have opportunities for future clinical research, but with limitations concerning the mass range of proteins. This setup is a powerful tool because detecting differences in cells with specific spatial coordinates, combined with the identification of these specific candidates by LC-MS/MS make it possible to merge biomarker discovery and histopathology. Regarding targeted analysis, only non-modified peptides are applicable for high quality absolute quantification, making the development of MRM methods even more difficult than it is these days using fresh material.

In conclusion, while considerable progress has been achieved, analysis of FFPE tissue proteome is still a major challenge. Using FFPE tissue in future proteome biomarker studies has potential, if more standardized methods will be applied and appropriate data-analysis software is available. 


\section{Figures}

Figure 1: Overview of different analytical platforms used in FFPE proteomics.

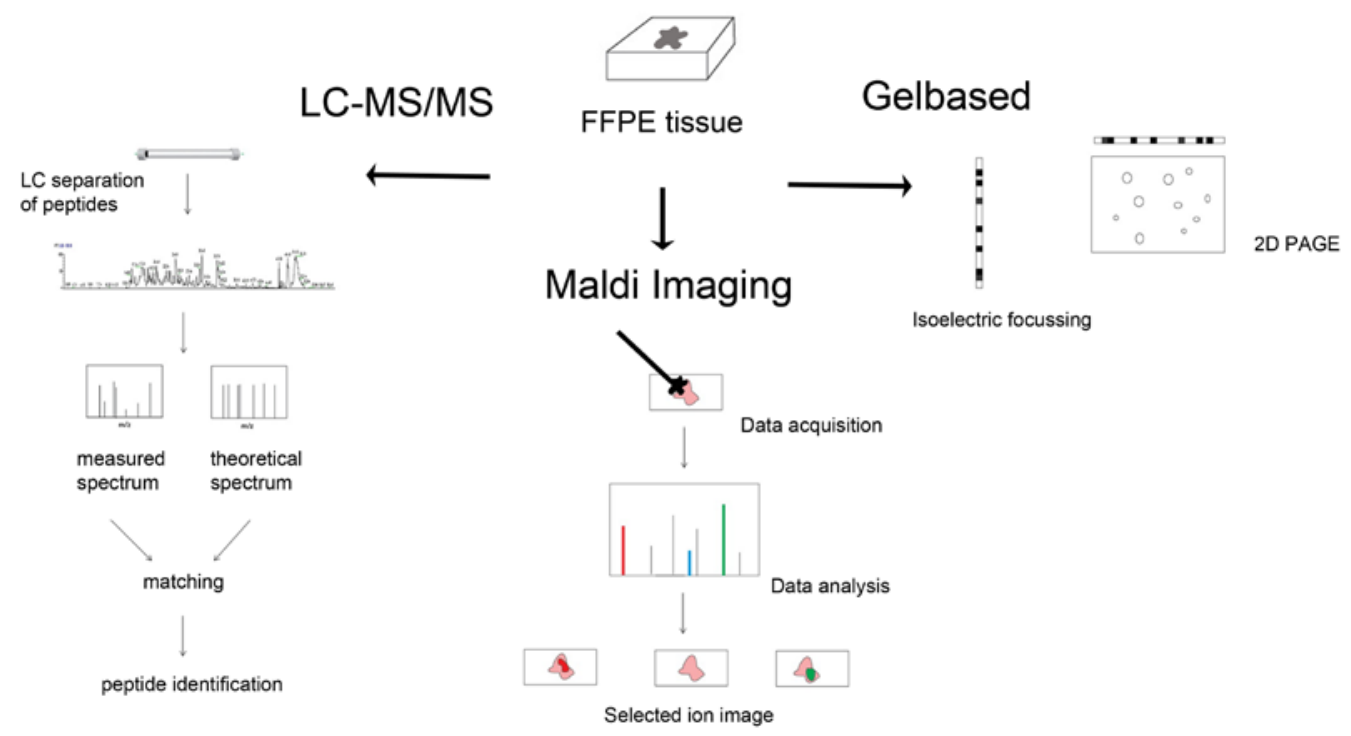

Tables

Table 1 : Compositions of different protein extraction buffers.

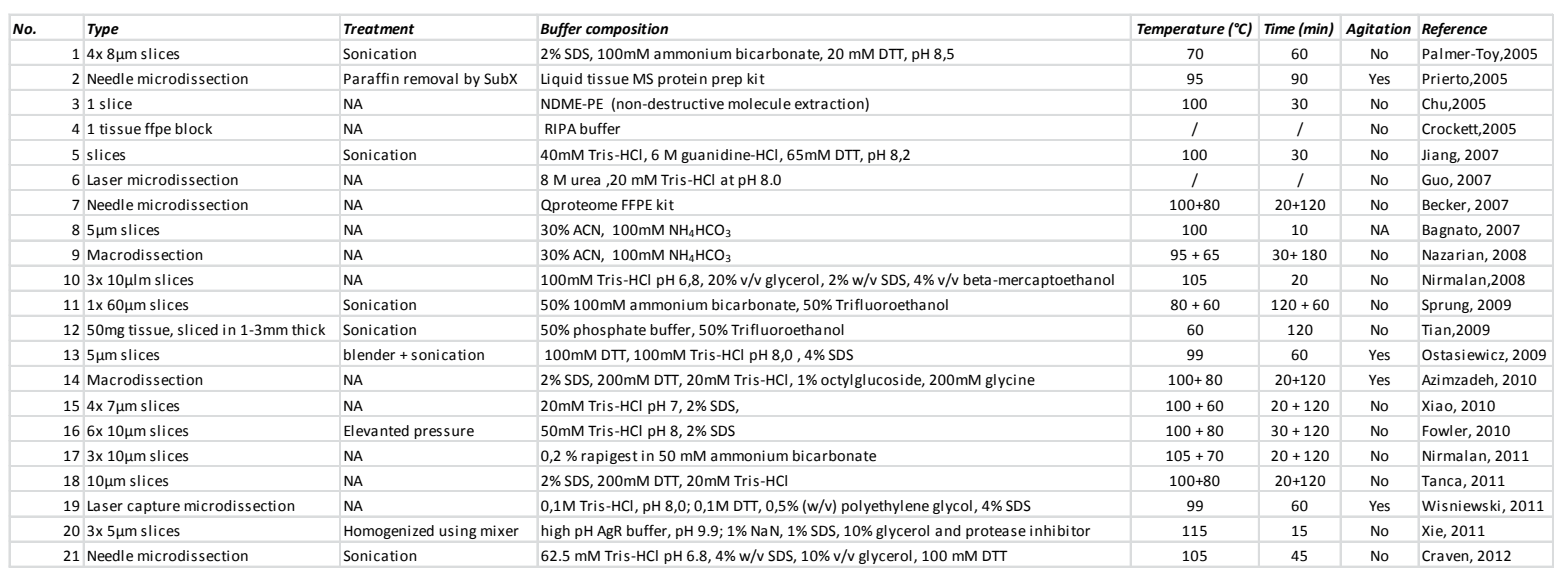


Table 2 : Overview of human clinical studies using FFPE proteomic approaches.

\begin{tabular}{|c|c|c|c|c|c|c|c|c|}
\hline & & Reference & Clinical tissue & \# samples & \# proteins identified & Analytical platform & Separation & Quantification \\
\hline \multicolumn{9}{|c|}{ Shotgun } \\
\hline & & Craven, 2012 & Kidney & 16 & $2516^{\S}$ & LC-MS/MS ; LTQ & RP-C18 & Spectral counting \\
\hline & & Naidoo, 2012 & Pancreas & 55 & 1504 & LC-MS/MS ; LTQ & $\mathrm{SCX}+\mathrm{RP}-\mathrm{C} 18$ & Spectral counting \\
\hline & & Nakatani, 2012 & Kidney & 20 & 170 & LC-MS/MS ; Q-TOF & NA & iTRAQ \\
\hline & & Wisnewski, 2012 & Colon & NA & 9502 & LC-MS/MS; Q exactive & MED-FASP-SAX + RP-C18 & Label-free, MaxQuant \\
\hline & 5 & Bateman, 2011 & Breast & 25 & $9437 *$ & LC-MS/MS ; LTQ & RP-C18 & Spectral counting \\
\hline & 6 & Donadio, 2011 & Parathyroid tissue & 5 & 163 & LC-MS/MS; LTQ Orbitrap XL & RP-C18 & None \\
\hline & 7 & Tanca, 2011 & Lung & 6 & 666 & GeLC-MS/MS ; Q-TOF & RP-C18 & Spectral counting \\
\hline & 8 & Wisnewski, 2011 & Colon & NA & 4419 & LC-MS/MS; LTQ Orbitrap XL & FASP-SAX + RP-C18 & Label-free, MaxQuant \\
\hline & 9 & Kawamura, 2010 & Lung & 13 & 649 & LC-MS/MS ; LTQ & RP-C18 & Spectral counting \\
\hline & 10 & Rezaul, 2010 & Melanoma & 1 tissue block & 935 & LC-MS/MS ; LTQ & NA & None \\
\hline & 11 ? & Xiao, 2010 & Pharynx & 40 & 730 & LC-MS/MS; Q-TOF & $\mathrm{SCX}+\mathrm{RP}-\mathrm{C} 18$ & iTRAQ \\
\hline & 12 & Huang, 2009 & Melanoma & 24 & 555 & LC-MS/MS ; LTQ & RP-C18 & Chromatographic peak intensity \\
\hline & 13 & Negishi, 2009 & Tongue & 20 & 25018 peaks $* *$ & LC-MS/MS ; Q-TOF & 2DICAL & 2DICAL \\
\hline & 14 & Nazarian, 2008 & Brainstem & 2 & 188 & LC-MS/MS ; LTQ & RP-C18 & Isotope labeling \\
\hline & 15 & Perroud, 2009 & Kidney & 50 & 777 & LC-MS/MS ; LTQ & RP-C18 & Spectral counting \\
\hline & 16 & Cheung, 2008 & Pancreas & 1 tissue block & 523 & LC-MS/MS ; LTQ & RP-C18 & None \\
\hline & 17. & Jain, 2008 & Oral HPV lesions & 10 & 114 & LC-MS/MS ; MALDI & RP-C18 & iTRAQ \\
\hline & 18 & Patel, 2008 & Head and neck & 16 & $391,866,729,676^{* * *}$ & LC-MS/MS ;LTQ & RP-C18 & Spectral counting \\
\hline & 19 & Ronci, 2008 & Breast & NA & $70,22,59 * * * *$ & LC-MS/MS ; Q-TOF & RP-C18 & None \\
\hline & 20 & Bagnato, 2007 & Coronary vessels & 35 & 710 & LC-MS/MS ; LTQ & RP-C18 & Spectral counting \\
\hline & 21 & Guo, 2007 & Glioblastoma & 1 tissue block & 2733 & LC-MS/MS ; LTQ & RP-C18 & None \\
\hline & 22 & Shi, 2006 & Kidney & 4 & 1830 & LC-MS/MS ; LTQ & $\mathrm{CIEF}+\mathrm{RP}-\mathrm{C} 18$ & None \\
\hline & 23 & Crockett, 2005 & Lymphoma & 1 tissue block & 324 & LC-MS/MS ; LCQ & RP-C18 & None \\
\hline & 24 & Hood, 2005 & Prostate & 2 & 1858 & LC-MS/MS ; LTQ or LIT-FT-ICR & RP-C18 & Isotope labeling \\
\hline & 25 & Palmer-Toy,2005 & Ear & 1 & 123 & LC-MS/MS ; LCQ Deca XP plus & RP-C19 & None \\
\hline & 26 & Prieto, 2005 & Colon & 1 & 350 & LC-MS/MS ; LTQ & RP-C18 & None \\
\hline & & & & & & & & \\
\hline \multicolumn{9}{|c|}{ MS Imaging } \\
\hline & 27 & Lazova, 2012 & Melanoma & 114 & 5 differential peptides & Maldi-MSI & None & None \\
\hline & 28 & Morgan, 2012 & Kidney & 70 & e signature of 7 and $12 \mathrm{pe}$ & $\in$ Maldi-MSI; Autoflex Speed & None & None \\
\hline & 29 & Morita, 2009 & Stomach & 9 & 4 cancer specific peptides & s Maldi-MSI; Qstar XL & None & None \\
\hline & 30 & Groseclose, 2008 & Lung & $50+10$ & 50 & Maldi-MSI & None & None \\
\hline \multicolumn{9}{|c|}{ Gelbased } \\
\hline & 31 & Tanca, 2012 & Stomach + Lung & NA & NA & Gelbased & 2D gel & 2D DIGE \\
\hline & 32 & Ono, 2009 & Cervix & 21 & 9 & Gelbased+ LC-MS/MS: LCQ & 2D gel & 2D DIGE \\
\hline \multirow{2}{*}{\multicolumn{9}{|c|}{ Targetted }} \\
\hline & & & & & & & & \\
\hline & 33 & Sprung, 2012 & Kidney, breast & NA & 1982 protein groups & LC-MS/MS ; LTQ XL & RP-C18 & MRM \\
\hline & 34 & Gamez-Pozo, 2011 & Lung + kidney & $3+3$ & 49 phospho $+154 / 42$ pho: & :LC-MS/MS ; LTQ-Orbitrap XL & $\mathrm{IMAC}+\mathrm{RP}-\mathrm{C} 18$ & Phospho + MRM \\
\hline & 35 & Güzel, 2011 & Placenta & 10 & 141 & LC-MS/MS ; QTRAP & RP-C18 & MRM \\
\hline & 36 & DeSouza, 2010 & Endometrium & 25 & 17 targeted proteins & LC-MS/MS ; QTRAP & RP-C18 & MRM (mTRAQ) \\
\hline & 37 & Nishimura, 2010 & Lung & 27 & NA & LC-MS/MS ; QTRAP & RP-C18 & MRM \\
\hline & 38 & Hwang, 2007 & Prostate & 30 & 428 & LC-MS/MS ; LTQ & RP-C18 & MRM (AQUA) \\
\hline & & & & & & & & \\
\hline$\S$ & & \multicolumn{3}{|c|}{ Total number of unique proteins in all samples } & & & & \\
\hline * & & \multicolumn{3}{|c|}{ Sum of proteins after triplicate injections of 25 samples } & & & & \\
\hline ** & & \multicolumn{3}{|c|}{ Only number of peaks described } & & & & \\
\hline *** & & \multicolumn{3}{|c|}{ Number of unique proteins per category } & & & & \\
\hline **** & & \multirow{2}{*}{\multicolumn{3}{|c|}{ Number of unique proteins per protocol }} & & & & \\
\hline & & & & & & & & \\
\hline
\end{tabular}

\section{Acknowledgements}

The authors acknowledge the Flemish Institute for Technological Research (VITO), the Industrial Research Fund (KU Leuven) and the Institute for the Promotion of Innovation through Science and Technology in Flanders (IWT). 


\section{Conflict of interest statement}

The authors declare that they have no conflict of interest.

\section{References}

Addis MF, Tanca A, Pagnozzi D, Rocca S, Uzzau S (2009) 2-D PAGE and MS analysis of proteins from formalin-fixed, paraffin-embedded tissues. Proteomics 9 (18):4329-4339

Ahram M, Flaig MJ, Gillespie JW, Duray PH, Linehan WM, Ornstein DK, Niu S, Zhao Y, Petricoin EF, III, Emmert-Buck MR (2003) Evaluation of ethanol-fixed, paraffin-embedded tissues for proteomic applications. Proteomics 3 (4):413-421

Amstalden van Hove ER, Smith DF, Heeren RM (2010) A concise review of mass spectrometry imaging. JChromatogrA 1217 (25):3946-3954

Angel PM, Caprioli RM (2013) Matrix-Assisted Laser Desorption Ionization Imaging Mass Spectrometry: In Situ Molecular Mapping. Biochemistry

Azimzadeh O, Barjaktarovic Z, Aubele M, Calzada-Wack J, Sarioglu H, Atkinson MJ, Tapio S (2010) Formalin-fixed paraffin-embedded (FFPE) proteome analysis using gel-free and gel-based proteomics. Journal of proteome research 9 (9):4710-4720

Bagnato C, Thumar J, Mayya V, Hwang SI, Zebroski H, Claffey KP, Haudenschild C, Eng JK, Lundgren DH, Han DK (2007) Proteomics analysis of human coronary atherosclerotic plaque: a feasibility study of direct tissue proteomics by liquid chromatography and tandem mass spectrometry. Mol Cell Proteomics 6 (6):1088-1102

Balgley BM, Guo T, Zhao K, Fang X, Tavassoli FA, Lee CS (2009) Evaluation of archival time on shotgun proteomics of formalin-fixed and paraffin-embedded tissues. J Proteome Res 8 (2):917-925

Bateman NW, Sun M, Bhargava R, Hood BL, Darfler MM, Kovatich AJ, Hooke JA, Krizman DB, Conrads TP (2011) Differential proteomic analysis of late-stage and recurrent breast cancer from formalin-fixed paraffin-embedded tissues. J Proteome Res 10 (3):1323-1332

Becker KF, Mack H, Schott C, Hipp S, Rappl A, Piontek G, Hofler H (2008) Extraction of Phosphorylated Proteins from Formalin-Fixed Cancer Cells and Tissues. The open pathology journal 2:46-52.

Cheung W, Darfler MM, Alvarez H, Hood BL, Conrads TP, Habbe N, Krizman DB, Mollenhauer J, Feldmann G, Maitra A (2008) Application of a global proteomic approach to archival precursor lesions: deleted in malignant brain tumors 1 and tissue transglutaminase 2 are upregulated in pancreatic cancer precursors. Pancreatology 8 (6):608-616

Cornett DS, Reyzer ML, Chaurand P, Caprioli RM (2007) MALDI imaging mass spectrometry: molecular snapshots of biochemical systems. Nat Methods 4 (10):828-833

Craven RA, Cairns DA, Zougman A, Harnden P, Selby PJ, Banks RE (2012) Proteomic analysis of formalin-fixed paraffin-embedded renal tissue samples by label-free MS: Assessment of overall technical variability and the impact of block age. ProteomicsClinAppl

Crockett DK, Lin Z, Vaughn CP, Lim MS, Elenitoba-Johnson KS (2005) Identification of proteins from formalin-fixed paraffin-embedded cells by LC-MS/MS. Lab Invest 85 (11):1405-1415

DeSouza LV, Krakovska O, Darfler MM, Krizman DB, Romaschin AD, Colgan TJ, Siu KW (2010) mTRAQ-based quantification of potential endometrial carcinoma biomarkers from archived formalin-fixed paraffin-embedded tissues. Proteomics 10 (17):3108-3116

DeSouza LV, Taylor AM, Li W, Minkoff MS, Romaschin AD, Colgan TJ, Siu KW (2008) Multiple reaction monitoring of $\mathrm{mTRAQ}$-labeled peptides enables absolute quantification of 
endogenous levels of a potential cancer marker in cancerous and normal endometrial tissues. J Proteome Res 7 (8):3525-3534

Djidja MC, Francese S, Loadman PM, Sutton CW, Scriven P, Claude E, Snel MF, Franck J, Salzet M, Clench MR (2009) Detergent addition to tryptic digests and ion mobility separation prior to MS/MS improves peptide yield and protein identification for in situ proteomic investigation of frozen and formalin-fixed paraffin-embedded adenocarcinoma tissue sections. Proteomics 9 (10):2750-2763

Donadio E, Giusti L, Cetani F, Da VY, Ciregia F, Giannaccini G, Pardi E, Saponaro F, Torregrossa L, Basolo F, Marcocci C, Lucacchini A (2011) Evaluation of formalin-fixed paraffin-embedded tissues in the proteomic analysis of parathyroid glands. Proteome Sci 9 (1):29

Fowler CB, Chesnick IE, Moore CD, O'Leary TJ, Mason JT (2010) Elevated pressure improves the extraction and identification of proteins recovered from formalin-fixed, paraffin-embedded tissue surrogates. PloS one 5 (12):e14253.

Gamez-Pozo A, Sanchez-Navarro I, Calvo E, Diaz E, Miguel-Martin M, Lopez R, Agullo T, Camafeita E, Espinosa E, Lopez JA, Nistal M, Vara JA (2011) Protein phosphorylation analysis in archival clinical cancer samples by shotgun and targeted proteomics approaches. Mol Biosyst 7 (8):2368-2374

Goodwin RJ, Dungworth JC, Cobb SR, Pitt AR (2008) Time-dependent evolution of tissue markers by MALDI-MS imaging. Proteomics 8 (18):3801-3808

Groseclose MR, Massion PP, Chaurand P, Caprioli RM (2008) High-throughput proteomic analysis of formalin-fixed paraffin-embedded tissue microarrays using MALDI imaging mass spectrometry. Proteomics 8 (18):3715-3724

Guo T, Wang W, Rudnick PA, Song T, Li J, Zhuang Z, Weil RJ, DeVoe DL, Lee CS, Balgley BM (2007) Proteome analysis of microdissected formalin-fixed and paraffin-embedded tissue specimens. J Histochem Cytochem 55 (7):763-772

Gustafsson JO, Oehler MK, McColl SR, Hoffmann P (2010) Citric acid antigen retrieval (CAAR) for tryptic peptide imaging directly on archived formalin-fixed paraffin-embedded tissue. J Proteome Res 9 (9):4315-4328

Guzel C, Ursem NT, Dekker LJ, Derkx P, Joore J, van DE, Ligtvoet G, Steegers EA, Luider TM (2011) Multiple reaction monitoring assay for pre-eclampsia related calcyclin peptides in formalin fixed paraffin embedded placenta. J Proteome Res 10 (7):3274-3282

Hewitt SM, Lewis FA, Cao Y, Conrad RC, Cronin M, Danenberg KD, Goralski TJ, Langmore JP, Raja RG, Williams PM, Palma JF, Warrington JA (2008) Tissue handling and specimen preparation in surgical pathology: issues concerning the recovery of nucleic acids from formalin-fixed, paraffin-embedded tissue. Arch Pathol Lab Med 132 (12):1929-1935

Hood BL, Darfler MM, Guiel TG, Furusato B, Lucas DA, Ringeisen BR, Sesterhenn IA, Conrads TP, Veenstra TD, Krizman DB (2005) Proteomic analysis of formalin-fixed prostate cancer tissue. Mol Cell Proteomics 4 (11):1741-1753

Huang SK, Darfler MM, Nicholl MB, You J, Bemis KG, Tegeler TJ, Wang M, Wery JP, Chong KK, Nguyen L, Scolyer RA, Hoon DS (2009) LC/MS-based quantitative proteomic analysis of paraffin-embedded archival melanomas reveals potential proteomic biomarkers associated with metastasis. PLoSOne 4 (2):e4430

Hwang SI, Thumar J, Lundgren DH, Rezaul K, Mayya V, Wu L, Eng J, Wright ME, Han DK (2007) Direct cancer tissue proteomics: a method to identify candidate cancer biomarkers from formalin-fixed paraffin-embedded archival tissues. Oncogene 26 (1):65-76

Ikeda K, Monden T, Kanoh T, Tsujie M, Izawa H, Haba A, Ohnishi T, Sekimoto M, Tomita N, Shiozaki $H$, Monden $M$ (1998) Extraction and analysis of diagnostically useful proteins from formalin-fixed, paraffin-embedded tissue sections. J Histochem Cytochem 46 (3):397-403

Jain MR, Liu T, Hu J, Darfler M, Fitzhugh V, Rinaggio J, Li H (2008) Quantitative Proteomic Analysis of Formalin Fixed Paraffin Embedded Oral HPV Lesions from HIV Patients. Open Proteomics J 1:40-45 
Kawamura T, Nomura M, Tojo H, Fujii K, Hamasaki H, Mikami S, Bando $Y$, Kato $H$, Nishimura T (2010) Proteomic analysis of laser-microdissected paraffin-embedded tissues: (1) Stagerelated protein candidates upon non-metastatic lung adenocarcinoma. J Proteomics 73 (6):1089-1099

Klockenbusch C, O'Hara JE, Kast J (2012) Advancing formaldehyde cross-linking towards quantitative proteomic applications. Anal Bioanal Chem 404 (4):1057-1067

Klopfleisch R, Weiss AT, Gruber AD (2011) Excavation of a buried treasure--DNA, mRNA, miRNA and protein analysis in formalin fixed, paraffin embedded tissues. Histol Histopathol 26 (6):797810

Lange V, Picotti P, Domon B, Aebersold R (2008) Selected reaction monitoring for quantitative proteomics: a tutorial. Mol Syst Biol 4:222

Lazova R, Seeley EH, Keenan M, Gueorguieva R, Caprioli RM (2012) Imaging mass spectrometry--a new and promising method to differentiate Spitz nevi from Spitzoid malignant melanomas. Am J Dermatopathol 34 (1):82-90

Lemaire R, Desmons A, Tabet JC, Day R, Salzet M, Fournier I (2007) Direct analysis and MALDI imaging of formalin-fixed, paraffin-embedded tissue sections. J Proteome Res 6 (4):12951305

Li Z, Adams RM, Chourey K, Hurst GB, Hettich RL, Pan C (2012) Systematic comparison of label-free, metabolic labeling, and isobaric chemical labeling for quantitative proteomics on LTQ Orbitrap Velos. J Proteome Res 11 (3):1582-1590

Link AJ, Eng J, Schieltz DM, Carmack E, Mize GJ, Morris DR, Garvik BM, Yates JR, III (1999) Direct analysis of protein complexes using mass spectrometry. Nat Biotechnol 17 (7):676-682

McDonnell LA, Heeren RM, Andren PE, Stoeckli M, Corthals GL (2012) Going forward: Increasing the accessibility of imaging mass spectrometry. J Proteomics 75 (16):5113-5121

Metz B, Kersten GF, Baart GJ, de JA, Meiring $H$, ten HJ, van Steenbergen MJ, Hennink WE, Crommelin DJ, Jiskoot W (2006) Identification of formaldehyde-induced modifications in proteins: reactions with insulin. Bioconjug Chem 17 (3):815-822

Metz B, Kersten GF, Hoogerhout P, Brugghe HF, Timmermans HA, de JA, Meiring H, ten HJ, Hennink WE, Crommelin DJ, Jiskoot W (2004) Identification of formaldehyde-induced modifications in proteins: reactions with model peptides. J Biol Chem 279 (8):6235-6243

Minerva L, Ceulemans A, Baggerman G, Arckens L (2012) MALDI MS imaging as a tool for biomarker discovery: methodological challenges in a clinical setting. Proteomics Clin Appl 6 (1112):581-595

Morgan TM, Seeley EH, Fadare O, Caprioli RM, Clark PE (2013) Imaging the clear cell renal cell carcinoma proteome. J Urol 189 (3):1097-1103

Morita Y, Ikegami K, Goto-Inoue N, Hayasaka T, Zaima N, Tanaka H, Uehara T, Setoguchi T, Sakaguchi T, Igarashi $H$, Sugimura $H$, Setou $M$, Konno H (2010) Imaging mass spectrometry of gastric carcinoma in formalin-fixed paraffin-embedded tissue microarray. Cancer Sci 101 (1):267-273

Naidoo K, Jones R, Dmitrovic B, Wijesuriya N, Kocher H, Hart IR, Crnogorac-Jurcevic T (2012) Proteome of formalin-fixed paraffin-embedded pancreatic ductal adenocarcinoma and lymph node metastases. J Pathol 226 (5):756-763

Nakatani S, Wei M, Ishimura E, Kakehashi A, Mori K, Nishizawa Y, Inaba M, Wanibuchi H (2012) Proteome analysis of laser microdissected glomeruli from formalin-fixed paraffinembedded kidneys of autopsies of diabetic patients: nephronectin is associated with the development of diabetic glomerulosclerosis. Nephrol Dial Transplant 27 (5):1889-1897

Nazarian J, Santi M, Hathout Y, Macdonald TJ (2008) Protein profiling of formalin fixed paraffin embedded tissue: Identification of potential biomarkers for pediatric brainstem glioma. Proteomics Clin Appl 2 (6):915-924

Negishi A, Masuda M, Ono M, Honda K, Shitashige M, Satow R, Sakuma T, Kuwabara H, Nakanishi Y, Kanai Y, Omura K, Hirohashi S, Yamada T (2009) Quantitative proteomics using formalin- 
fixed paraffin-embedded tissues of oral squamous cell carcinoma. Cancer Sci 100 (9):16051611

Nirmalan NJ, Hughes C, Peng J, McKenna T, Langridge J, Cairns DA, Harnden P, Selby PJ, Banks RE (2011) Initial development and validation of a novel extraction method for quantitative mining of the formalin-fixed, paraffin-embedded tissue proteome for biomarker investigations. Journal of proteome research 10 (2):896-906.

Nirmalan NJ, Harnden P, Selby PJ, Banks RE (2008) Mining the archival formalin-fixed paraffinembedded tissue proteome: opportunities and challenges. Mol Biosyst 4 (7):712-720

Nishimura T, Nomura M, Tojo H, Hamasaki H, Fukuda T, Fujii K, Mikami S, Bando Y, Kato H (2010) Proteomic analysis of laser-microdissected paraffin-embedded tissues: (2) MRM assay for stage-related proteins upon non-metastatic lung adenocarcinoma. J Proteomics 73 (6):1100-1110

Ono A, Kumai T, Koizumi H, Nishikawa H, Kobayashi S, Tadokoro M (2009) Overexpression of heat shock protein 27 in squamous cell carcinoma of the uterine cervix: a proteomic analysis using archival formalin-fixed, paraffin-embedded tissues. Hum Pathol 40 (1):41-49

Ostasiewicz P, Zielinska DF, Mann M, Wisniewski JR (2010) Proteome, phosphoproteome, and Nglycoproteome are quantitatively preserved in formalin-fixed paraffin-embedded tissue and analyzable by high-resolution mass spectrometry. J Proteome Res 9 (7):3688-3700

Palmer-Toy DE, Krastins B, Sarracino DA, Nadol JB, Jr., Merchant SN (2005) Efficient method for the proteomic analysis of fixed and embedded tissues. J Proteome Res 4 (6):2404-2411

Patel V, Hood BL, Molinolo AA, Lee NH, Conrads TP, Braisted JC, Krizman DB, Veenstra TD, Gutkind JS (2008) Proteomic analysis of laser-captured paraffin-embedded tissues: a molecular portrait of head and neck cancer progression. Clin Cancer Res 14 (4):1002-1014

Perroud B, Ishimaru T, Borowsky AD, Weiss RH (2009) Grade-dependent proteomics characterization of kidney cancer. Mol Cell Proteomics 8 (5):971-985

Picotti P, Aebersold R (2012) Selected reaction monitoring-based proteomics: workflows, potential, pitfalls and future directions. Nat Methods 9 (6):555-566

Prieto DA, Hood BL, Darfler MM, Guiel TG, Lucas DA, Conrads TP, Veenstra TD, Krizman DB (2005) Liquid Tissue: proteomic profiling of formalin-fixed tissues. Biotechniques Suppl:32-35

Ralton LD, Murray GI (2011) The use of formalin fixed wax embedded tissue for proteomic analysis. J Clin Pathol 64 (4):297-302

Reimel BA, Pan S, May DH, Shaffer SA, Goodlett DR, McIntosh MW, Yerian LM, Bronner MP, Chen R, Brentnall TA (2009) Proteomics on Fixed Tissue Specimens - A Review. Curr Proteomics 6 (1):63-69

Rezaul K, Murphy M, Lundgren DH, Wilson L, Han DK (2010) Combined mass spectrometry- and immunohistochemistry-based approach to determine protein expression in archival melanoma--proof of principle. Pigment Cell Melanoma Res 23 (6):849-852

Ronci M, Bonanno E, Colantoni A, Pieroni L, Di IC, Spagnoli LG, Federici G, Urbani A (2008) Protein unlocking procedures of formalin-fixed paraffin-embedded tissues: application to MALDITOF imaging MS investigations. Proteomics 8 (18):3702-3714

Seeley EH, Caprioli RM (2011) MALDI imaging mass spectrometry of human tissue: method challenges and clinical perspectives. Trends Biotechnol 29 (3):136-143

Shi SR, Key ME, Kalra KL (1991) Antigen retrieval in formalin-fixed, paraffin-embedded tissues: an enhancement method for immunohistochemical staining based on microwave oven heating of tissue sections. J Histochem Cytochem 39 (6):741-748

Shi SR, Liu C, Balgley BM, Lee C, Taylor CR (2006) Protein extraction from formalin-fixed, paraffinembedded tissue sections: quality evaluation by mass spectrometry. J Histochem Cytochem 54 (6):739-743

Sprung RW, Martinez MA, Carpenter KL, Ham AJ, Washington MK, Arteaga CL, Sanders ME, Liebler DC (2012) Precision of Multiple Reaction Monitoring Mass Spectrometry Analysis of Formalin-Fixed, Paraffin-Embedded Tissue. J Proteome Res 
Sprung RW, Jr., Brock JW, Tanksley JP, Li M, Washington MK, Slebos RJ, Liebler DC (2009) Equivalence of protein inventories obtained from formalin-fixed paraffin-embedded and frozen tissue in multidimensional liquid chromatography-tandem mass spectrometry shotgun proteomic analysis. MolCell Proteomics 8 (8):1988-1998

Tanca A, Addis MF, Pagnozzi D, Cossu-Rocca P, Tonelli R, Falchi G, Eccher A, Roggio T, Fanciulli G, Uzzau S (2011a) Proteomic analysis of formalin-fixed, paraffin-embedded lung neuroendocrine tumor samples from hospital archives. J Proteomics 74 (3):359-370

Tanca A, Pagnozzi D, Addis MF (2012a) Setting proteins free: progresses and achievements in proteomics of formalin-fixed, paraffin-embedded tissues. Proteomics Clin Appl 6 (1-2):7-21

Tanca A, Pagnozzi D, Burrai GP, Polinas M, Uzzau S, Antuofermo E, Addis MF (2012b) Comparability of differential proteomics data generated from paired archival fresh-frozen and formalinfixed samples by GeLC-MS/MS and spectral counting. J Proteomics 77:561-576

Tanca A, Pagnozzi D, Falchi G, Tonelli R, Rocca S, Roggio T, Uzzau S, Addis MF (2011b) Application of 2-D DIGE to formalin-fixed, paraffin-embedded tissues. Proteomics 11 (5):1005-1011

Tanca A, Pisanu S, Biosa G, Pagnozzi D, Antuofermo E, Burrai GP, Canzonieri V, Cossu-Rocca P, De RV, Eccher A, Fanciulli G, Rocca S, Uzzau S, Addis MF (2012c) Application of 2-D DIGE to formalin-fixed diseased tissue samples from hospital repositories: results from four case studies. Proteomics Clin Appl

Tian Y, Gurley K, Meany DL, Kemp CJ, Zhang H (2009) N-linked glycoproteomic analysis of formalinfixed and paraffin-embedded tissues. Journal of proteome research 8 (4):1657-1662.

Toews J, Rogalski JC, Clark TJ, Kast J (2008) Mass spectrometric identification of formaldehydeinduced peptide modifications under in vivo protein cross-linking conditions. Anal Chim Acta 618 (2):168-183

Walch A, Rauser S, Deininger SO, Hofler H (2008) MALDI imaging mass spectrometry for direct tissue analysis: a new frontier for molecular histology. Histochem Cell Biol 130 (3):421-434

Washburn MP, Wolters D, Yates JR, III (2001) Large-scale analysis of the yeast proteome by multidimensional protein identification technology. Nat Biotechnol 19 (3):242-247

Wisniewski JR, Dus K, Mann M (2012) Proteomic workflow for analysis of archival formalin-fixed and paraffin-embedded clinical samples to a depth of 10000 proteins. Proteomics Clin Appl

Wisniewski JR, Ostasiewicz P, Mann M (2011) High recovery FASP applied to the proteomic analysis of microdissected formalin fixed paraffin embedded cancer tissues retrieves known colon cancer markers. J Proteome Res 10 (7):3040-3049

Wu Q, Yuan H, Zhang L, Zhang $Y$ (2012) Recent advances on multidimensional liquid chromatography-mass spectrometry for proteomics: from qualitative to quantitative analysis--a review. Anal Chim Acta 731:1-10

Xiao Z, Li G, Chen Y, Li M, Peng F, Li C, Li F, Yu Y, Ouyang Y, Xiao Z, Chen Z (2010) Quantitative proteomic analysis of formalin-fixed and paraffin-embedded nasopharyngeal carcinoma using iTRAQ labeling, two-dimensional liquid chromatography, and tandem mass spectrometry. J Histochem Cytochem 58 (6):517-527

Xie R, Chung JY, Ylaya K, Williams RL, Guerrero N, Nakatsuka N, Badie C, Hewitt SM (2011) Factors influencing the degradation of archival formalin-fixed paraffin-embedded tissue sections. The journal of histochemistry and cytochemistry : official journal of the Histochemistry Society 59 (4):356-365.

Ye X, Blonder J, Veenstra TD (2009) Targeted proteomics for validation of biomarkers in clinical samples. Brief Funct Genomic Proteomic 8 (2):126-135 